\title{
Associations between digital dermatitis lesion grades in dairy cattle and the quantities of four Treponema species
}

\author{
Caroline Beninger ${ }^{1}$, Syed Ali Naqvi ${ }^{1}$, Sohail Naushad ${ }^{1}$, Karin Orsel ${ }^{1}$, Chris Luby², Hooman Derakhshani \\ Ehsan Khafipour ${ }^{3}$ and Jeroen De Buck ${ }^{1 *}$ (D)
}

\begin{abstract}
Digital dermatitis (DD) presents as painful, ulcerative or proliferative lesions that lead to bovine lameness affecting economic efficiency and animal welfare. Although DD etiological agent(s) have not been established, it is widely accepted that DD is a polymicrobial disease significantly associated with species of Treponema and the non-linear disease progression may be attributed to interactions among infecting bacteria. We postulated the morphological changes associated with DD lesion grades are related to interactions among infecting species of Treponema. We developed a novel species-specific qPCR that can identify the absolute abundance of the four of the most common species of Treponema in DD, T. phagedenis, T. medium, T. pedis and T. denticola, in a single reaction. We found species abundance and the number of distinct Treponema species present is higher in active, ulcerative lesions than in healing lesions, chronic lesions, and DD-free skin. Treponema spp. were present in both DD-free skin and M3 lesions following treatment with oxytetracycline. We have also found positive correlations among T. phagedenis, T. medium and T. pedis indicating they are significantly more likely to be found together than apart and their absolute quantities tend to increase together, a relationship which is not present with $T$. denticola. Further, we found Treponema, particularly viable T. denticola, in lesions 5 days post treatment with oxytetracycline (M3). Our findings suggest that pathogenicity may be closely associated with Treponema abundance, particularly T. phagedenis, T. medium and T. pedis, and interactions among them, independent of $T$. denticola. Our results provide a novel, consistent method to identify species of Treponema within DD lesions and associate Treponema spp. and abundance with morphological changes related to host pathogenicity.
\end{abstract}

\section{Introduction}

Globally, digital dermatitis (DD) is a leading cause of infectious lameness in dairy cattle [1]. Digital dermatitis is mainly found on the plantar aspect of a cow's foot, presenting as an ulcerative or proliferative lesion along the coronary band, affecting the area between the heel bulbs [1]. A review paper on lameness prevalence studies throughout the world between 1993 and 2014 found that between 25 and $55 \%$ of dairy cattle are clinically lame on average, $15-22 \%$ of cows have one or more DD

\footnotetext{
*Correspondence: jdebuck@ucalgary.ca

${ }^{1}$ Department of Production Animal Health, Faculty of Veterinary

Medicine, University of Calgary, Calgary, AB, Canada

Full list of author information is available at the end of the article
}

lesions, and up to $94 \%$ of herds have DD [2-4]. The infectious, ulcerative or proliferative lesions caused by DD contribute to animal welfare concerns and significant economic losses due to premature culling, milk loss, decreased fertility, and treatment costs $[4,5]$. The complex polymicrobial nature of the disease and non-linear lesion progression have contributed to the inability to determine the etiological agent(s) for DD despite significant economic incentives [6, 7]. Species of Treponema, fastidious, anaerobic bacteria, are consistently isolated from DD lesions in polytreponemal communities [810]. While there is considerable evidence implicating Treponema spp. as causative agents in DD, Koch's postulates have yet to be satisfied [1,10-12]. It has been more than 40 years since DD was first described, and despite

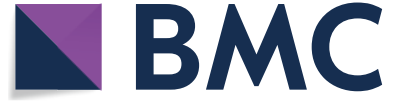

(c) The Author(s) 2018. This article is distributed under the terms of the Creative Commons Attribution 4.0 International License (http://creativecommons.org/licenses/by/4.0/), which permits unrestricted use, distribution, and reproduction in any medium, provided you give appropriate credit to the original author(s) and the source, provide a link to the Creative Commons license, and indicate if changes were made. The Creative Commons Public Domain Dedication waiver (http://creativecommons.org/ publicdomain/zero/1.0/) applies to the data made available in this article, unless otherwise stated. 
efforts to prevent the disease with improved hygiene practices, regular footbaths, and bedding modifications, effective treatments that eliminate the disease from herds have not been identified [5, 13]. Furthermore, treatments for DD with $100 \%$ sustained cure rate have not been identified and treated lesions are prone to relapsing into active disease states or remaining chronic [14-16].

Methods to simultaneously identify multiple species of Treponema within DD lesions are currently limited to $16 \mathrm{~S}$ rRNA gene sequencing-based microbiome approaches which cannot reliably resolve species composition due to partial $16 \mathrm{~S}$ gene coverage, limited complete genomes available and many unidentified Treponema species. Treponema phylotypes are based on culture independent methods such as 16S rRNA and/or flaB2 sequencing and 16S-23S intergenic spacer region sequence analysis which distinguishes phylotypes based on size $[9,15,17$, 18]. Phylotypes are defined as clusters of Treponema in which the $16 \mathrm{~S}$ rRNA sequence differs by approximately $2 \%$ from a known Treponema spp. and species within a cluster must share $\geq 99 \%$ sequence similarity with other members of their cluster [1]. Phylotypes are mostly named based upon their sequence similarity to human Treponema isolates, primarily periodontal disease isolates [10]. Because researchers use different sequences for clustering isolates are clustered differently, impacting prevalence data between studies $[9,15,17,18]$.

Previous studies have associated Treponema abundance with lesion severity and temporal progression, demonstrating that the composition of species present changes considerably as lesions progress [19]. The microbial community present within DD lesions has been shown to correlate with morphological changes of lesions; however, while this relationship is significantly correlated with the 5 grade Iowa DD scoring system, which primarily differs from $\mathrm{M}$-grade scoring by having a temporal aspect to lesion progression and adding 2 early grade lesions, it has not been examined using the M-scoring system [19]. Therefore, current evidence suggests Treponema spp. and bacterial community composition may be related to morphological changes associated with grades of DD lesion grades.

There is increasing evidence implicating multiple species of Treponema in DD etiology and pathogenicity; however, there is a knowledge deficit regarding potential interactions among species present [20]. Based on current evidence, we postulate a relationship between the Treponema spp. present and DD lesion grades and/or DD progression that may be facilitated by synergistic interactions among species. The aims of this study were to examine the correlations among Treponema spp. within and between lesion grades. For this purpose, a novel species-specific qPCR was developed to identify species present, their abundance, and relative proportions in DD lesions. Identifying correlations between species of Treponema present in DD lesions may provide essential targets for vaccines and treatment development by indicating species synergies required for enhanced virulence or pathogenicity.

\section{Materials and methods \\ Farm and animal details}

Farms were selected based on previous DD prevalence information and geographical location, facilitated by two certified Alberta hoof trimmers. Samples were collected from 10 Alberta farms and one abattoir between Calgary and Ponoka throughout a 1.5-year period. Additionally, 10 samples were collected from a Saskatchewan dairy herd that has been a closed farm for over 50 years with a reported zero DD prevalence (Rayner Dairy Research and Teaching Facility, University of Saskatchewan). All animal use was approved under protocol \#AC16-0070, by the University of Calgary Veterinary Services Animal Care Committee (VSACC) under the guidance of the Canadian Council on Animal Care (CCAC) prior to the onset of the study.

\section{DD scoring}

Digital dermatitis lesions were identified on farm in the trimming chute according to the $\mathrm{M}$-grade scoring system and limited to lesions located between the heel bulbs [13, 21]. Briefly, M1 and M2 lesions were ulcerative masses along the coronary band, distinguished by a lesion smaller or larger than $2 \mathrm{~cm}$, respectively [21]. Due to the difficulty in characterizing M3 lesions without treatment history, we classified M3 lesions as active lesions approximately 5 days after antibiotic treatment. M4 lesions were raised, hyperkeratotic, and proliferative, with papilliform projections and M4.1 lesions were M4 lesions with an active ulcerative area.

\section{Biopsy}

Identified lesions were scrubbed twice using a chlorohexidine scrub followed by a $70 \%$ ethanol wipe while animals were in a no-tilt trimming chute to remove debris and clean the area. Lidocaine Neat $(2-3 \mathrm{~mL})$ (Lidocaine $\mathrm{HCl}$ 2\%, DIN 00712884, Zoetis Canada Inc., Kirkland, QC, Canada) was administered subcutaneously with a $20 \mathrm{~g}$ needle. Small, $4 \mathrm{~mm}$ biopsy punches with a maximum coring depth of $7 \mathrm{~mm}$ (Standard Disposable Biopsy Punch, Miltex, Integra Life Sciences Corporation, York, PA, USA) were taken from various DD lesion grades from dairy cows on 10 Alberta farms and DD-free feet from the abattoir and 1 closed herd in Saskatchewan $(N=142)$. For lesions with an ulcerative focus, M1, M2, and M4.1, the biopsies were collected from the active area. "M3 
biopsies" were collected from M2 lesions, identified, treated and wrapped by a certified hoof trimmer, 5 days after antibiotic treatment with soluble tetracycline250 powder $(250 \mathrm{mg} / \mathrm{g}$ ) (DIN 0052777, Vetoquinol, Lavaltrie, QC, Canada). Samples between the heel bulbs from non-infected hooves $(N=21)$ were collected from culled Holstein cows at the abattoir less than $2 \mathrm{~h}$ post-mortem. Two biopsies from DD-infected feet at the abattoir were taken to ensure Treponema could be cultured from tissue samples collected post-mortem, if present. Biopsies were immediately placed into semi-solid anaerobic transport media (ATM) (Anaerobe Systems, Morgan Hill, CA, USA) with the interior portion inserted first to avoid contaminating the biopsies with microorganisms from the outer skin and transported at room temperature. The transport medium, ATM, contains sodium thioglycolate and cysteine reducing agents to sustain viability of anaerobic microbes during transport with minimal multiplication. Biopsy samples from within Alberta were transported to the lab and processed within $8 \mathrm{~h}$ of sampling and biopsies from Saskatchewan were processed within $24 \mathrm{~h}$ of sampling.

\section{Culture and isolation}

In the anaerobic cabinet (Bactron3000, Sheldon Manufacturing, Inc., Cornelius, OR, USA) $\left(25 \% \mathrm{CO}_{2}, 5 \% \mathrm{H}_{2}\right.$, balance nitrogen), on a sterile petri dish with sterile tissue forceps and a no. 10 scalpel blade, the outermost portion of the epidermis was removed from the biopsies and discarded. The remaining interior portion was sectioned longitudinally into 4 fragments of approximately equal size and macerated. Biopsy fragments for DNA extraction and storage were inoculated into Tris-EDTA buffer (TE) $(\mathrm{pH}=8.0)$ and OTEB $+20 \%$ glycerol, and stored at $-20{ }^{\circ} \mathrm{C}$ and $-80{ }^{\circ} \mathrm{C}$, respectively. Biopsy fragments for culture were inoculated into oral treponeme enrichment broth (OTEB) (Anaerobe Systems, Morgan Hill, CA, USA) with $5 \%$ enrofloxacin ( $\geq 98 \%$ HPLC, powder, Sigma-Aldrich, Burlington, MA, USA) dissolved in DMSO, $10 \%$ rifampicin ( $\geq 97 \%$ HPLC, powder, SigmaAldrich, Burlington, MA, USA) dissolved in DMSO, and $10 \%$ equal parts bovine and rabbit serum (Gibco, Life Technologies, New Zealand and USA, respectively) (OTEBSER) and MTGE media supplemented with $5 \%$ enrofloxacin and 10\% rifampicin by spread plating (MTGEER) (Anaerobe Systems, Morgan Hill, CA, USA). Biopsy fragments were inserted into MTGE medium using a no. 10 scalpel blade and sterile forceps to ensure the fragments were laterally and distally surrounded by agar. MTGEER plates were wrapped in parafilm and incubated for 7 days at $37^{\circ} \mathrm{C}$. Motile bacteria (those that migrate away from the biopsy) forming white-translucent, small, and irregular colony morphologies on the surface of, or embedded within, the agar were selected from each plate and subcultured into $1 \mathrm{~mL}$ of OTEBSER in $1.5 \mathrm{~mL}$ Eppendorf tubes and incubated for up to 10 days at $37{ }^{\circ} \mathrm{C}$ in the anaerobic cabinet. Subculture purity, absence of contaminating non-treponeme bacteria, was assessed using dark field or differential interference contrast (DIC) microscopy based on Treponema morphological characteristics (thin corkscrew-shaped bacteria between 0.1 and $0.4 \mu \mathrm{m}$ in width and $4-15 \mu \mathrm{m}$ in length, and rotational movement about the longitudinal axis) [22]. Subcultures were screened for T. phagedenis, T. medium, T. pedis and T. denticola with an in-house species-specific multiplex PCR outlined below. Cultures containing other bacteria in addition to Treponema were spread plated onto MTGEER, incubated for 7 days at $37^{\circ} \mathrm{C}$ in the anaerobic cabinet, and subcultured into OTEBSER as above until contaminant free Treponema cultures were obtained.

Macerated biopsy fragments in $200 \mu \mathrm{L}$ of TE were weighed twice and averaged prior to freezing and DNA extraction. DNA from macerated biopsy fragments in TE was extracted using DNeasy Blood and Tissue Extraction Kit according to the manufacturer's recommendations (Qiagen, Hilden, Germany). DNA was eluted into nuclease-free ultra pure water and stored at $-20^{\circ} \mathrm{C}$.

\section{qPCR design and optimization}

A 4-plex Treponema species-specific qPCR was designed to quantify four Treponema spp. and their absolute quantities in bovine tissue samples. Genomic DNA extracted from 7 DD Treponema isolates, which were identified as T. phagedenis, T. pedis, and T. medium, from the University of Wisconsin, provided by Dr. Döpfer, were sequenced, assembled and annotated as described previously [23]. For species-specific gene identification, an inhouse database containing newly sequenced Treponema genomes and representative genome sequences from all known bacterial species was constructed from NCBI, EZBioCloud, PATRIC, and JGI-IMG [24-27]. Potential species-specific genes were identified according to Naushad et al. [28]. Briefly, BLASTn searches were conducted on all open reading rrames (ORFs) from T. pedis, T. phagedenis, T. medium and T. denticola against the inhouse database. The ORFs which were detected in a single Treponema species and were not found in any other representative bacterial species were considered potential species-specific genes. The specificity of potential species-specific genes was validated after blast searching against the NCBI nr database. The ORFs detected in all known strains of each species were considered unique genes. Unique genes selected for each Treponema species were CP004120 for T. pedis, WP_002698807.1 for T. phagedenis, WP_016523385.1 for T. medium, and 
EGC77593.1 for T. denticola. Based on BLAST results and available complete reference genomes for the four Treponema species, we assume there is a single speciesspecific gene copy found in each species. Primer and probes against species-specific genes were designed with idtDNA PrimerQuest software (Integrated DNA Technologies, 2017). Primer sequences were designed with an equal melting temperature $\left(\mathrm{T}_{\mathrm{m}}\right)$ of approximately $60{ }^{\circ} \mathrm{C}$ and probes had a Tm of approximately $70{ }^{\circ} \mathrm{C}$ or $10{ }^{\circ} \mathrm{C}$ above the primer $\mathrm{Tm}$. The length of the qPCR products was set between 90 and $150 \mathrm{bp}$. Runs of consecutive nucleotides and GC clamps were avoided, and GC content was below $60 \%$ in the primers and probes (TaqMan Probe Design, Premier Biosoft). Primer and probe sequences that met the above criteria were analyzed for hairpins, self-dimers, and hetero-dimers using PriDimerCheking 0.1.0 software [29] to avoid secondary structure between primers and probes. Only sequences without hairpins at the Tm of the sequence and $\Delta \mathrm{G}$ values, which indicate energetic favourability of a given structural conformation, more positive than $-9 \mathrm{kcal} / \mathrm{mol}$ were selected.

Conventional PCR using the genes identified above were designed to verify species composition to validate the qPCR and culture composition (Table 1). Final forward and reverse primer concentrations were $0.3 \mu \mathrm{M}$ in $23 \mu \mathrm{L}$ of TopTaq mastermix ( $12.5 \mu \mathrm{L} /$ reaction), nuclease free water, $(6.5 \mu \mathrm{L} /$ reactions $)$ and coral load $(2.5 \mu \mathrm{L} /$ reaction) (TopTaq ${ }^{\mathrm{TM}}$ Master Mix Kit, Qiagen) and $2 \mu \mathrm{L}$ of template DNA. Reaction conditions were $95^{\circ} \mathrm{C}$ for $5 \mathrm{~min}$, $\left(94{ }^{\circ} \mathrm{C} 30 \mathrm{~s}, 57^{\circ} \mathrm{C}\right.$ for $\left.30 \mathrm{~s} 72{ }^{\circ} \mathrm{C} 40 \mathrm{~s}\right) \times 35,72{ }^{\circ} \mathrm{C} 5 \mathrm{~min}$.

To multiplex the qPCR, the likelihood of all combinations of primer and probe sequences to form secondary structure were calculated using PriDimerCheking 0.1.0 software (Table 1) [29]. Sequences with the lowest alignment score and the most positive $\Delta G$ value were selected. Each species was designated a distinct fluorophore with minimal overlap in the absorption and emission spectra based on the Spectral Overlay Tool (Biosearch Technologies, Novato, CA, USA). All qPCR reactions were performed in TaqMan ${ }^{\circledR}$ Fast Advanced Master Mix (Applied Biosystems ${ }^{\circledR}$, ThermoFisher, Foster City, CA, USA). The annealing temperature of the qPCR reaction was optimized using a temperature gradient $3{ }^{\circ} \mathrm{C}$ above and below the mean annealing temperature of all primer and probe sets (CFX96 Touch $^{\mathrm{TM}}$ System, Bio-Rad Laboratories, Inc., Hercules, CA, USA). The optimal annealing temperature was the highest temperature at which the lowest quantitation cycle (cq) value is found for all reactions (59$60.4{ }^{\circ} \mathrm{C}$ ); if the optimal annealing temperature was not the same in all reactions, the temperature was averaged without weighting $\left(59.6^{\circ} \mathrm{C}\right)$. The primer and probe concentrations were optimized by varying the forward and reverse primer and probe concentrations $(50,100,200$, 400,600 , and $800 \mathrm{nM}$ ); the optimal concentration was the lowest concentration for both primers that achieved the lowest cq value and $>98 \%$ assay efficiency $(1 \mu \mathrm{M})$. Assay efficiency was calculated using $E=10^{(-1 / \text { slope })}$, where slope is the slope of the standard curve of each assay (Figure 1) (CFX Manager ${ }^{\mathrm{TM}}$ Software, Bio-Rad Laboratories, Inc., Hercules, CA, USA). The reactions were optimized as single reactions and then as multiplex reactions. Single and multiplex reactions were run in parallel and conditions were accepted when cq values differed by less than 0.5 between single and multiplex reactions and efficiency remained $\geq 95 \%$. Final qPCR conditions for multiplex reactions was: $50^{\circ} \mathrm{C} 2 \mathrm{~min}, 95^{\circ} \mathrm{C} 20 \mathrm{~s},\left(95^{\circ} \mathrm{C} 10 \mathrm{~s}, 59.6^{\circ} \mathrm{C}\right.$ $50 \mathrm{~s}) \times 39,72^{\circ} \mathrm{C} 5 \mathrm{~min}$.

\section{qPCR absolute quantification}

To relate the cq value to gene copy number, clones containing the species-specific genes of interest were generated. Species-specific PCR products were purified using Qiagen QIAquick PCR Purification Kit and TOPO cloned into commercially available NEB $5-\alpha \mathrm{F}^{\prime} \mathrm{I}^{\mathrm{q}}$ chemically competent Escherichia coli cells according to the

Table 1 Primer and probe sequences for Treponema species-specific fourplex qPCR and conventional PCR

\begin{tabular}{|c|c|c|c|c|}
\hline Species & Forward primer (sense) & Probe (sense) & Reverse Primer (antisense) & Size (bp) \\
\hline T. denticola ${ }^{\mathrm{a}}$ & GGAAACTTAGGAATTCGATATGTAG & AGCATACAGCGATTATAACAAAGCCCTCGA & CCTTCTTTAGTTTCTTTGTGAGG & 113 \\
\hline T. medium ${ }^{\mathrm{a}}$ & AAAGCGCTACGAATCCTAAG & TGCACCCTTGTTTACTACTGCACAGCC & ATCATTACCCGTCCACAAAG & 119 \\
\hline T. phagedenis ${ }^{\mathrm{a}}$ & CCCGCAGGAAGGTATAATC & AATCCGCCTACGACTGCGATACCA & CACAGCTGTTGTGGTATTAAG & 90 \\
\hline T. pedis ${ }^{\mathrm{a}}$ & ACACCGATTGTACTGAATGA & ACTACACGTGGAGTACCGAATGCT & CCACGAGCTTTCTACAGATT & 118 \\
\hline T. denticola ${ }^{b}$ & AGGAATGGCCTTTGAACCCGCA & & CCGATGAACCCGTATCTTCACCGA & 458 \\
\hline T. medium ${ }^{b}$ & GGAACAGGCAGCCGCATTGGAT & & CCGCCCATGTGAGGCTTGTGAT & 515 \\
\hline T. phagedenis ${ }^{\mathrm{b}}$ & TCCGCCTACGACTGCGATACCA & & CGGAACTGTCACAACTGGCGGA & 785 \\
\hline T. pedis ${ }^{\mathrm{b}}$ & TGGATGTTACGGAAGAGACACCGA & & TGCCCCACTCTTACAAGTTCATCCCA & 295 \\
\hline
\end{tabular}

\footnotetext{
a Primer and probe sequences for species of Treponema for qPCR.
}

b Conventional PCR primer sequences for Treponema species. 


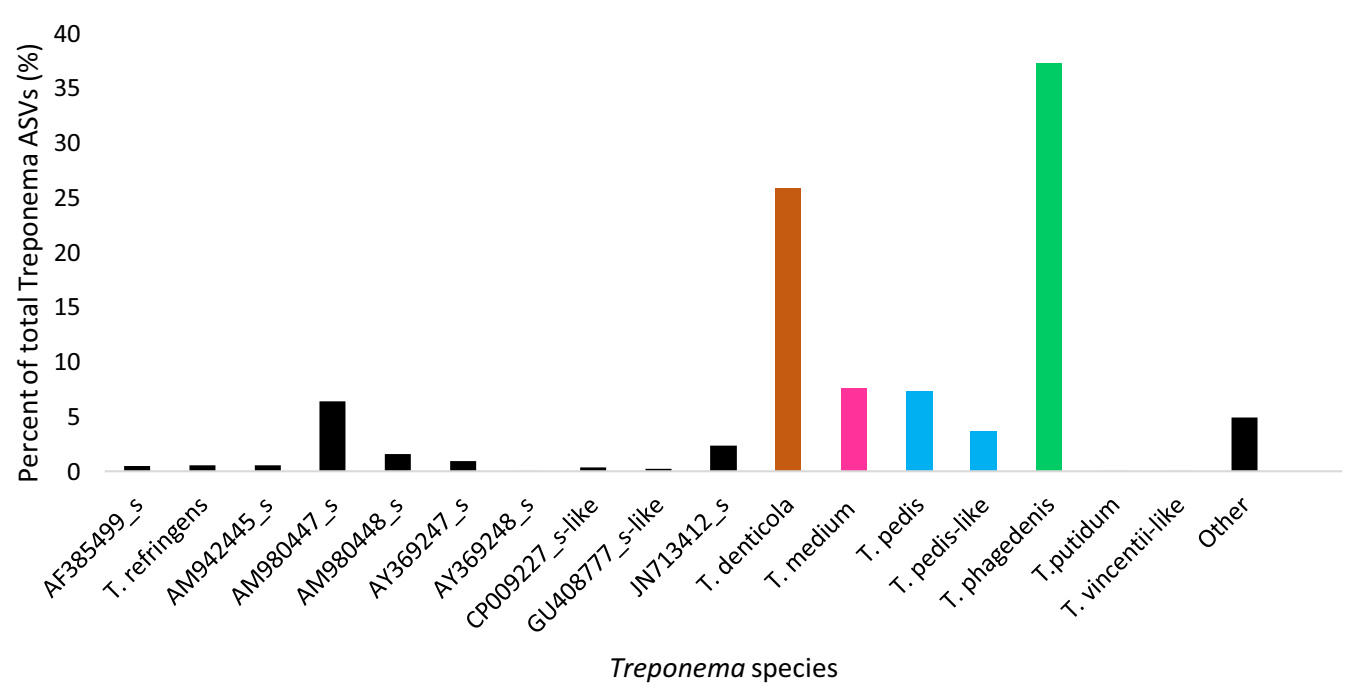

Figure 1 Microbiome analysis indicating ASVs designated to the genus Treponema from DNA directly extracted from DD-infected biopsies $(\boldsymbol{N}=\mathbf{1 6})$. Accession numbers are included for Treponema spp. that could not be identified at the species level but were identified as members of the genus Treponema.

manufacturer's recommendations (TOPO ${ }^{\circledR}$ TA Cloning ${ }^{\circledR}$ Kit, Invitrogen, Carlsbad, CA, USA). Transformants were identified following blue-white screening by direct colony PCR using the primer sets in Table 1.

Absolute quantification of qPCR products was determined using a standard curve of known concentrations of the above-mentioned clones and the corresponding cq values. The amount of plasmid DNA following purification was determined using a Qubit Fluorometer $\left(\mathrm{Qubit}^{\circledR}\right.$ dsDNA HS Assay Kits, Life Technologies, Carlsbad, CA, USA). To ensure the calculated copy number is representative of the number of species in a biopsy sample and quantify sensitivity, we performed spiking experiments. To spike biopsy samples, known numbers of Treponema cells, roughly estimated using a Neubauer counting chamber with DIC microscopy at $40 \times$ magnification, were serially diluted $10^{0}-10^{-7}$ and spiked into lesion-free biopsy samples $(N=10)$ that did not contain the species of interest according to our species-specific PCR and qPCR. Treponema cultures of various species composition were grown for 7 days in OTEB in the anaerobic cabinet as described above. Cultures were pelleted by centrifugation at $4500 \times g$ at $8{ }^{\circ} \mathrm{C}$ for $20 \mathrm{~min}$, the supernatant removed, and the pellet resuspended in $200 \mu \mathrm{L}$ of PBS. The biopsy samples were macerated as described above to mimic biopsy processing procedures and spiked with serially diluted Treponema samples or an equal volume of PBS as a negative control. Each culture serial dilution was divided into two samples; one was spiked into qPCR-negative bovine tissue while the other was directly extracted without bovine tissue. DNA from cultures, spiked tissues, and negative control tissues were extracted using Qiagen DNeasy Blood and Tissue kit (Qiagen). The results from the spiked tissue samples were compared to an equal amount of the culture used to inoculate the tissue sample. The efficiency of the multiplex reactions was determined by converting the resulting cq values to copy numbers to determine if there was significant DNA loss at any point in the experimental procedure and accurately quantify the number of Treponema species per gram of biopsy tissue.

\section{Microbiome analysis}

Microbiome analysis of the 16S rRNA V1-V2 hypervariable region was performed using Illumina MiSeq at the University of Manitoba with DNA extracted from 16 biopsy samples and T. denticola, T. medium, and $T$. phagedenis ATCC culture DNA provided by Dr. Luby of the University of Saskatchewan (ATCC \#33520, 700293, 27087, respectively). Microbiome analysis data, including lesion grade, ASVs, farm ID, cow ID, and which leg the biopsy was taken from, are included (Additional file 1). UNOISE2 algorithm was selected to cluster sequences based on $100 \%$ sequence similarity to create amplicon sequence variants (ASVs) [30] whereas UPARSE created OTUs de novo based on $97 \%$ sequence similarity [31]. Representative ASV reads were then aligned to the GreenGenes (release May 2013) [32] database to assign taxonomies at the genus level. ASVs identified as Treponema and Spirochaeates were blasted using the NCBI database and EzBioCloud to assign species level 
classifications (>97\% identity) and ASVs between 93 and 97\% identities were grouped into "other".

\section{qPCR validation}

Microbiome analysis data was used to validate the qPCR by testing samples determined to contain T. denticola, $T$. phagedenis, T. pedis and/or T. medium and samples without the above species to ensure agreement. In the case that multiple ASVs corresponded to a single Treponema species, any hits for that species were included as a positive result. Positive and negative predictive values were calculated using microbiome analysis data as the standard for comparison [33]. Specificity of the qPCR products were verified by a single band at the respective base pair length using $1.4 \%$ agarose gel electrophoresis run at $120 \mathrm{~V}$ for $15 \mathrm{~min}$. Species-specific gene primers were used to amplify gene fragments from 3 Treponema cultures of biopsies originating from geographically distinct farm locations and Sanger sequenced to ensure desired gene product was amplified. Global pairwise alignments with free end gaps were generated between all available online and in-house copies of species-specific genes and sequenced fragments in Geneious (10.1.3, Biomatters Ltd., 2017) based on dynamic programming [34]. We assumed high similarity between sequences and used $93 \%$ similarity cost matrix $(5.0 /-9.02)$, gap open penalty of 12 and gap extension penalty of 3.

\section{Statistical analysis}

Statistical analyses were conducted in R v.3.4.2 (R Core Team 2017) and a $P$ value $<0.05$ was considered statistically significant. As multiple samples were collected from each herd, the degree of clustering at the herd-level was first determined using a multivariate linear regression with herd included as a predictor. Based on the limited magnitude of variance explained by herd as a predictor for each species (approximately 5\%), herd effects were not included in subsequent models. Additionally, stratification by herd resulted in a significant loss of power since within-herd sample sizes were much smaller than the total sample size, making it impossible to investigate the main variable of interest-lesion grade. Prior to statistical analysis, qPCR copy numbers were standardized by unit of weight using the mean sample weight and the corresponding DNA yield in $\mathrm{ng}(18.15 \mathrm{mg} / \mathrm{ng})$.

Pairwise associations between species presence were assessed using bivariate logistic regression in the statistical package "zeligverese" [35] for each pair of species. The outcome for each model was the presence or absence of both species from each pair, with lesion grade as a predictor. The bivariate logistic regression allowed us to determine the probability of finding a given pair of species together relative to the probability of isolating them separately. Pairwise inter-species correlations of the copy number per mg of tissue were calculated using Pearson's correlation coefficient.

Prior to analysis a natural logarithm transformation was used to normalize the data as observations were strongly skewed to the right. A multinomial regression using the "nnet" package [36] was used to assess differences between lesion grades in the combinations of species that were found. Lesion grade was used as the outcome for this regression, with each distinct combination of species isolated being used as a categorical predictor. This model allowed for pairwise comparisons between combinations of species to determine the most common combinations found within each lesion grade and overall. To compare differences between qPCR copynumbers per $\mathrm{mg}$ of species between lesion grades, we selected a multivariate normal regression using the "lm" function in base R. A multivariate normal regression allowed for the unbiased assessment of statistical differences by accounting for the inter-species correlations.

\section{Results}

In total, 142 biopsies were collected from 132 cows; most cows sampled twice were from the slaughterhouse with 6 cows on farms sampled on separate occasions or from lesions on different feet (Table 2).

\section{Microbiome analysis data}

In total, 56 distinct ASVs from 16 biopsy samples belonged to the genus Treponema when UPARSE (31 OTUs) and UNOISE2 (55 ASVs) data were combined. When assigned species identification, $T$. phagedenis, $T$. denticola, T. medium, T. pedis, and AM980447_s, which is most closely related to $T$. denticola with $94 \%$ identity, were the most common species of Treponema representing 37.3, 25.9, 7.6, 7.3 and $6.4 \%$ of total Treponema ASVs, respectively (Figure 1). Only 6 Treponema ASVs could be identified at the species level (>97\% identity), $T$. refringens and $T$. putidum in addition to the above four species which represented $79 \%$ of the Treponema present. Ultimately, T. refringens and T. putidum made up $0.61 \%$ and $0.09 \%$ of the Treponema species populations, respectively (Figure 1). All other Treponema spp. (>90\% sequence similarity) could not be identified (Figure 1) and are reported as the closest hit or accession number on EzBioCloud. Sequences that could not be clustered into an accession number (less than $97 \%$ similarity to an accession number) were grouped together as "other", which represented 5\% of the Treponema spp. populations (Figure 1). Treponema species identified made up 79\% of Treponema spp. in biopsy samples and $21 \%$ could not be identified at the species level. 
Table 2 Representation of all biopsies collected and cultured from 10 Alberta farms, 1 Saskatchewan farm, and an Alberta slaughterhouse, by lesion grade

\begin{tabular}{|c|c|c|c|c|c|c|c|}
\hline \multirow[t]{2}{*}{ Farm } & \multirow[t]{2}{*}{ No. cows } & \multicolumn{6}{|c|}{ Lesion grade } \\
\hline & & Mo & M1 & M2 & M3 & M4 & M4.1 \\
\hline 1 & 4 & 0 & 0 & 3 & 0 & 0 & 1 \\
\hline 2 & 20 & 0 & 2 & 6 & 0 & 6 & 10 \\
\hline 3 & 7 & 0 & 1 & 1 & 0 & 1 & 4 \\
\hline 4 & 12 & 0 & 6 & 5 & 0 & 0 & 1 \\
\hline 5 & 22 & 0 & 0 & 11 & 13 & 0 & 0 \\
\hline 6 & 6 & 0 & 1 & 2 & 0 & 0 & 3 \\
\hline 7 & 15 & 0 & 4 & 7 & 0 & 1 & 4 \\
\hline 8 & 3 & 0 & 0 & 0 & 0 & 2 & 1 \\
\hline 9 & 8 & 0 & 0 & 0 & 0 & 1 & 7 \\
\hline 10 & 26 & 0 & 1 & 5 & 0 & 3 & 6 \\
\hline $11^{\mathrm{S}}$ & 10 & 10 & 0 & 0 & 0 & 0 & 0 \\
\hline Abattoir & 9 & 21 & 1 & 0 & 0 & 1 & 0 \\
\hline Total & 142 & 31 & 16 & 40 & 13 & 15 & 37 \\
\hline
\end{tabular}

S Indicates Saskatchewan farm.

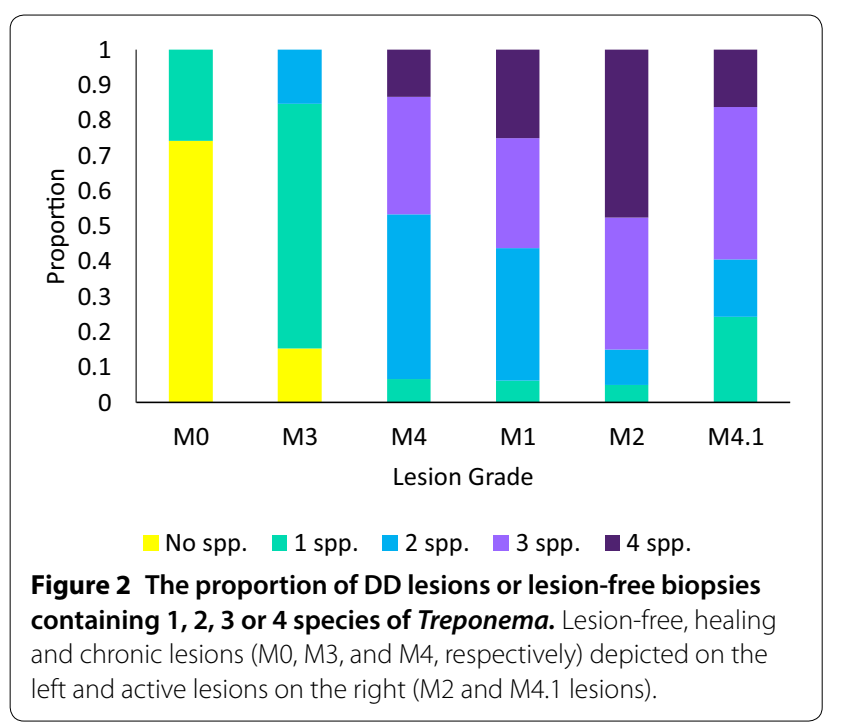

\section{qPCR validation}

The sequenced gene fragments for $T$. phagedenis (785 bp) had $99.6 \%$ pairwise identity among Alberta isolates $(N=4)$ and $95.3 \%$ to the 3 species-specific gene sequences from which the primers were designed. The sequenced gene fragments for T. medium (515 bp) had 99.8\% pairwise identity among Alberta isolates $(N=3)$ and $94.5 \%$ identical to 2 species-specific gene sequences from which the primers were designed. The sequenced gene fragments for T. pedis (295 bp) had $93.7 \%$ pairwise identity to each other $(N=4)$ and $97.5 \%$ pairwise identity to 5 species-specific gene sequences from which the primers were designed. The sequenced gene fragments for $T$. denticola (458 bp) had $90.3 \%$ pairwise identity among Alberta isolates $(N=3)$ and $94.7 \%$ pairwise identity to 5 species-specific gene sequences from which the primers were designed.

Microbiome analysis data was analyzed to verify the specificity of the 4-plex qPCR reaction (Figure 1). Regardless of the algorithm used to identify species, UPARSE or UNOISE2, the qPCR specificity was equal. The qPCR for T. phagedenis, T. medium, T. pedis and T. denticola agreed with microbiome analysis results $100 \%$, $100 \%, 93.4 \%$, and $87.5 \%$ of the time, respectively, based on 16 samples. In the case of T. pedis, there was a single false negative in the qPCR, where the sample had $0.015 \%$ of its total ASVs determined to be T. pedis by microbiome analysis. However, there were 2 and 3 distinct ASVs in UPARSE and UNOISE2, respectively and the ASV with $100 \%$ identity to T. pedis was 0 in both algorithms. There was a false positive and a false negative for T. denticola. The false negative comprised of 15 hits $(0.06 \%$ of sample ASVs) and the false positive was just above the threshold ( $\mathrm{cq}=40)$ of detection ( $\mathrm{cq}=39.72)$. Sensitivity for T. phagedenis, T. medium, T. pedis and T. denticola were $100 \%, 100 \%, 93.0 \%$ and $93.3 \%$, respectively. Specificity for T. phagedenis, T. medium, T. pedis were $100 \%$ whereas $T$. denticola was $67 \%$ due to a single false positive. Our spiking experiment indicated the average DNA extraction and qPCR detection efficiency was between 96 and $99.5 \%$ for all species (Table 3).

\section{Number of Treponema species cultured or detected in DD lesions}

There were Treponema cultured or detected by qPCR from $100 \%$ of active (M1 lesions, M2 lesions, and M4.1 
Table 3 Spiking experiment results indicating the efficiency of Treponema DNA extraction and detection, with and without bovine tissue, using species-specific qPCR

\begin{tabular}{|c|c|c|c|c|c|c|c|}
\hline \multirow[t]{2}{*}{ Species } & \multicolumn{2}{|c|}{$\begin{array}{l}1 \\
\text { Mean count }\end{array}$} & \multicolumn{2}{|c|}{$\begin{array}{l}1 / 10 \\
\text { Mean count }\end{array}$} & \multicolumn{2}{|c|}{$\begin{array}{l}1 / 100 \\
\text { Mean count }\end{array}$} & \multirow{2}{*}{$\begin{array}{l}\text { Average } \\
\text { efficiency } \\
(\%)\end{array}$} \\
\hline & Culture & Tissue (count/mg) & Culture & Tissue (count/mg) & Culture & Tissue (count/mg) & \\
\hline T.phagedenis & $2.32 \times 10^{8}$ & $4.22 \times 10^{7}$ & $2.78 \times 10^{8}$ & $3.32 \times 10^{7}$ & $2.26 \times 10^{7}$ & $1.04 \times 10^{7}$ & 99.5 \\
\hline T. medium & $8.72 \times 10^{7}$ & $9.76 \times 10^{5}$ & $1.40 \times 10^{8}$ & $6.80 \times 10^{6}$ & $1.22 \times 10^{7}$ & $9.45 \times 10^{5}$ & 98.8 \\
\hline T.pedis & $1.41 \times 10^{9}$ & $7.93 \times 10^{7}$ & $6.85 \times 10^{5}$ & $7.80 \times 10^{4}$ & $9.31 \times 10^{4}$ & $5.25 \times 10^{3}$ & 96.0 \\
\hline T. denticola & $2.58 \times 10^{6}$ & $1.24 \times 10^{5}$ & $4.14 \times 10^{5}$ & $8.36 \times 10^{4}$ & $1.23 \times 10^{5}$ & $1.59 \times 10^{4}$ & 98.2 \\
\hline
\end{tabular}

${ }^{a}$ Cq values were converted into gene copy numbers and the mean count between samples without tissue was compared to the mean count with tissue samples of tenfold serially diluted Treponema cultures.

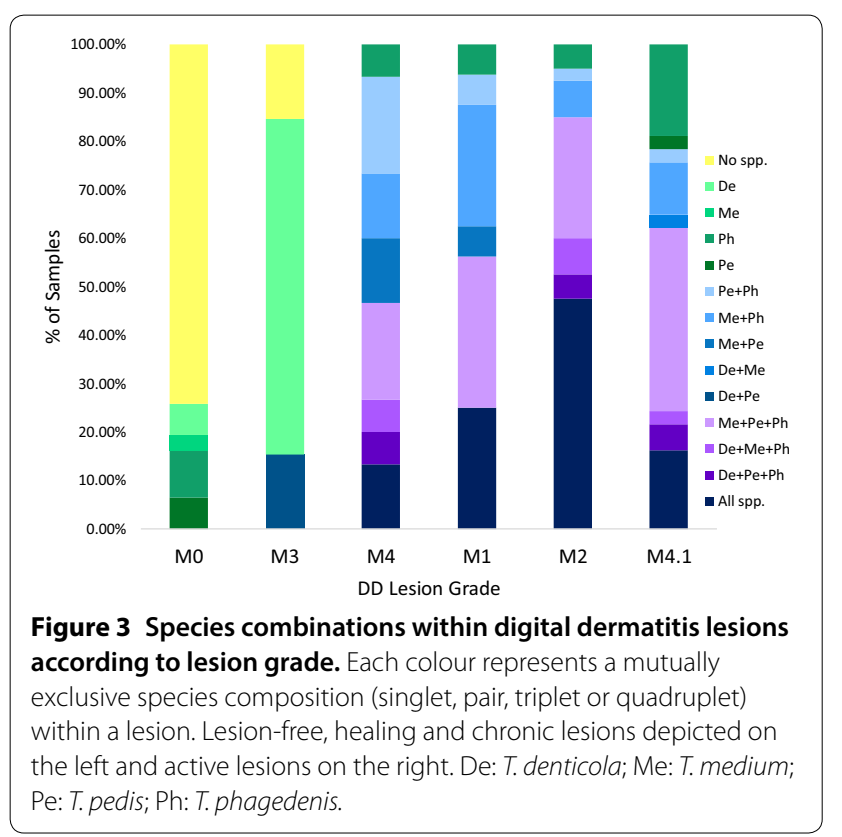

lesions) and chronic DD lesions (M4 lesions). Unless otherwise specified, direct detection refers to Treponema detected by qPCR directly from biopsy tissue before culture. A small proportion (15.4\%) of healing/M3 lesions did not contain any of the 4 Treponema species or two species of Treponema (15.4\%) and most (69.2\%) contained 1 species of Treponema (Figure 2). The majority of M1 lesions and M4 lesions contained 2 species of Treponema (37.5\% and $46.7 \%$, respectively), followed by 3 species (31.3\% and $33 \%$, respectively), 4 species ( $25 \%$ and $13.3 \%$, respectively), and a very small proportion containing 1 species (6.25\% and 6.67\%, respectively) (Figure 2). Conversely, the majority of M2 lesions and M4.1 lesions contained 3 (30\% and $32.4 \%$, respectively) or 4 species of Treponema (47.5\%, $16.4 \%$, respectively). Similarly, $10 \%$ of M2 lesions and $10.8 \%$ of M4.1 lesions contain only 2 species while only $5 \%$ of M2 lesions and $18.9 \%$ of M4.1 lesions contain only 1 species of Treponema (Figure 2). The majority of M0 samples did not contain any of the 4 species of Treponema listed above (74.2\%) (Table 4); M0 biopsies that contained species of Treponema typically contained only 1 and never exceeded 2 of the examined Treponema species (v 3). Treponema could not be isolated from M0 samples despite being initially detected visually within the sample tissue by dark field microscopy and qPCR. However, Treponema were detected and cultured from all DD-infected biopsies $(N=2)$ collected from the abattoir indicating Treponema could be cultured from tissue collected post-mortem.

Table 4 Identification of four Treponema species $(N=142)$ according to lesion grade by culture and species-specific PCR

\begin{tabular}{|c|c|c|c|c|c|c|}
\hline \multirow[t]{2}{*}{ Lesion grade } & \multicolumn{5}{|c|}{ No. samples with species present (\%) } & \multirow[t]{2}{*}{ Total } \\
\hline & T.phagedenis & T. medium & T.pedis & T. denticola & No spp. & \\
\hline Mo & $3(9.7)$ & $1(3.2)$ & $2(6.5)$ & $2(6.5)$ & $23(74.2)$ & $31(100)$ \\
\hline M1 & $15(93.8)$ & $14(87.5)$ & $11(68.8)$ & $4(25)$ & $0(0)$ & $16(100)$ \\
\hline M2 & $40(100)$ & $35(87.5)$ & $32(80)$ & $24(60)$ & $0(0)$ & $40(100)$ \\
\hline M3 & $0(0)$ & $0(0)$ & $2(15.4)$ & $13(100)$ & $2(15.4)$ & $13(100)$ \\
\hline M4 & $13(86.7)$ & $10(66.7)$ & $11(73.3)$ & $4(26.7)$ & $0(0)$ & $15(100)$ \\
\hline M4.1 & $35(94.6)$ & $26(70.3)$ & $24(64.9)$ & $10(27)$ & $0(0)$ & $37(100)$ \\
\hline Total & $106(69.7)$ & $86(56.6)$ & $82(53.9)$ & $55(36.2)$ & 25 (16.4) & $152(100)$ \\
\hline
\end{tabular}


Culture and qPCR detection results indicated that, irrespective of lesion grade, T. phagedenis was the most commonly identified and isolated species of Treponema, followed by T. medium, T. pedis and T. denticola (Table 4). Multiple species of Treponema were detected in M3 samples by qPCR before culture; however, the only viable species detected in M3 lesions 5 days post-treatment with tetracycline was T. denticola which was detected in $100 \%$ of M3 cultures (Table 4). Culture and PCR results from isolated Treponema showed at least two cultures containing one or more unidentified species by positive microscopic detection and negative PCR results for T. denticola, T. medium, T. pedis and T. phagedenis. The two unknown samples were sequenced with two universal primer sets, resulting in sequence identity between 79 and $94 \%$ to known species of Treponema; most closely related to T. medium.

The presence or absence of each of the four species of Treponema was analyzed by lesion grade. Within lesion grades, T. phagedenis was consistently the most commonly identified species except for M3 lesions in which T. denticola was the most commonly identified species. Within M1 lesions and M2 lesions, T. medium was the second most commonly identified species of the four Treponema species, present in $87.5 \%$ of samples. Within M4 lesions, $T$. pedis is the second most common species (73.3\%) followed by T. medium (66.7\%) (Table 4). Overall, T. denticola was the least common species identified (36.2\%) (Table 4).

\section{Direct detection of Treponema in biopsies}

Despite T. phagedenis being the most common of the four Treponema species to be found in M0 samples, it was significantly (3.3-3.6 times) more likely that $T$. phagedenis would be present in M1 lesions, M2 lesions, and M4.1 lesions than M0 samples $(P=0.004,0.0001$, and 0.0001 , respectively) (Table 6$)$. Interestingly there were no significant differences between $T$. phagedenis presence or absence in M4 lesions, M3 lesions and M0 samples $(P>0.99)$. It was 2-3.7 times more likely to find $T$. pedis in M1 lesions, M2 lesions, M4 lesions, and M4.1 lesions than M0 samples $(P=0.03,0.001,0.04$, and 0.08$)$. It was significantly more likely to find $T$. pedis (2.4 times) in M2 lesions than M3 lesions $(P=0.048)$ and there were no significant differences between M0 samples and M3 lesions for T. pedis presence or absence (Table 5). Surprisingly, there were no significant differences in the presence or absence of T. medium in any lesion grades $(P>0.6)$. M2 lesions, M3 lesions, M4 lesions and M4.1 lesions were significantly more likely to contain $T$. denticola than M0 samples $(P=0.005,0.001,0.037$, and 0.018 , respectively). Further, $T$. denticola was significantly more likely to be found in M3 lesions and M2 lesions than M1 lesions $(P=0.015$ and 0.056$)$ and there were no significant
Table 5 Difference between lesion grades in log odds of finding Treponema species across lesion grades assuming no relationships or dependence among species

\begin{tabular}{|c|c|c|c|c|}
\hline \multirow{2}{*}{$\begin{array}{l}\text { Lesion } \\
\text { Comparison }{ }^{a}\end{array}$} & \multicolumn{4}{|l|}{ Species } \\
\hline & T. denticola & T. medium & T.pedis & T. phagedenis \\
\hline M0-M1 & -0.77 & -19.72 & $-2.55^{*}$ & $-3.28^{* *}$ \\
\hline M0-M2 & $-3.86^{* * *}$ & -19.80 & $-2.82^{* * *}$ & $-3.26^{* * *}$ \\
\hline M0-M3 & $-4.96^{* * *}$ & 0.00 & -0.37 & 16.49 \\
\hline M0-M4 & -3.95 & -20.26 & $-3.69^{*}$ & -20.65 \\
\hline M0-M4.1 & $-3.52^{* *}$ & -18.94 & $-1.99^{\ddagger}$ & $-3.64^{* * *}$ \\
\hline M1-M2 & $-3.09^{\ddagger}$ & -0.08 & -0.27 & 0.03 \\
\hline M1-M3 & $-4.19^{* *}$ & 19.72 & 2.17 & 19.77 \\
\hline M1-M4 & -3.18 & -0.54 & -1.14 & -17.36 \\
\hline M1-M4.1 & -2.75 & 0.78 & 0.56 & -0.35 \\
\hline M2-M3 & -1.10 & 19.80 & $2.44^{*}$ & 19.74 \\
\hline$M 2-M 4$ & -0.09 & -0.46 & -0.87 & -17.39 \\
\hline M2-M4.1 & 0.34 & 0.86 & 0.82 & -0.38 \\
\hline M3-M4 & 1.01 & -20.26 & -3.31 & -37.13 \\
\hline M3-M4.1 & 1.44 & -18.94 & -1.62 & -20.12 \\
\hline M4-M4.1 & 0.43 & 1.32 & 1.70 & 17.01 \\
\hline
\end{tabular}

${ }^{a}$ Negative value indicates lesion grade on the left is $\operatorname{exp(log~odds)~times~less~}$ likely to contain the given species; positive value indicates lesion grade on the left is exp(log odds) times more likely to contain the given species.

${ }^{*} P<0.05$, ${ }^{* *} P<0.01,{ }^{* * *} P<0.001,{ }^{\ddagger} 0.051<P<0.90$.

differences between the presence of $T$. denticola between M0 samples and M1 lesions $(P>0.99)$ (Table 5$)$.

\section{Treponema species composition: direct detection in biopsies}

A multinomial analysis of the presence or absence of a species irrespective of lesion grade indicated significant relationships between T. phagedenis, T. pedis, and $T$. medium that were independent of $T$. denticola. Treponema phagedenis was 26.2 times more likely to be found with $T$. medium than without $(P=0.003)$ and T. medium was 7.5 times more likely to be found with $T$. pedis than without $(P<0.001)$. Interestingly, $T$. phagedenis is only 2.5 times more likely to be found with T. pedis than without; however, the latter result was only suggestive of a relationship and did not reach statistical significance $(P=0.10)$. Odds ratios for $T$. denticola in the presence of T. pedis, T. medium and T. phagedenis, were $1.75,0.99$ and 0.84 , respectively, but failed to reach significance ( $P=0.27,0.99$, and 0.78 , respectively).

The composition of Treponema species was analyzed between DD lesion grades to examine potential interactions among species. Overall, the most common species compositions were $T$. phagedenis, $T$ medium, and T. pedis $(\mathrm{Ph} / \mathrm{Me} / \mathrm{Pe})$ and $T$. phagedenis, $T$ medium, T. pedis, and T. denticola (Ph/Me/Pe/De) $(21.1 \%$ and $20.4 \%$, 
respectively) (Figure 3). Within lesions, all four species of Treponema was the most common species composition within M2 lesions (47.5\%). However, in M1 lesions T. medium, T. phagedenis, and T. pedis $(\mathrm{Ph} / \mathrm{Me} / \mathrm{Pe})$ was the most common species composition (31.3\% of M1 lesions), followed by all four species ( $\mathrm{Ph} / \mathrm{Me} / \mathrm{Pe} / \mathrm{D})$ and $T$. medium and $T$. phagedenis together which were equally likely at $25 \%$ of M1 lesions each (Figure 3 ). Similarly, Ph/ $\mathrm{Me} / \mathrm{Pe}$ and T. phagedenis and T. pedis were equally likely species composition in M4 lesions comprising 20\% (to a total of $40 \%$ ) of M4 biopsies collected. $\mathrm{Ph} / \mathrm{Me} / \mathrm{Pe}$ was the most common species compositions in M4.1 lesions comprising $37.8 \%$ of biopsies followed by T. phagedenis alone and all 4 species ( $\mathrm{Ph} / \mathrm{Me} / \mathrm{Pe} / \mathrm{De})$ comprising $18.9 \%$ and $16.2 \%$ of samples, respectively. In M3 lesions, T. denticola alone was the most common species composition

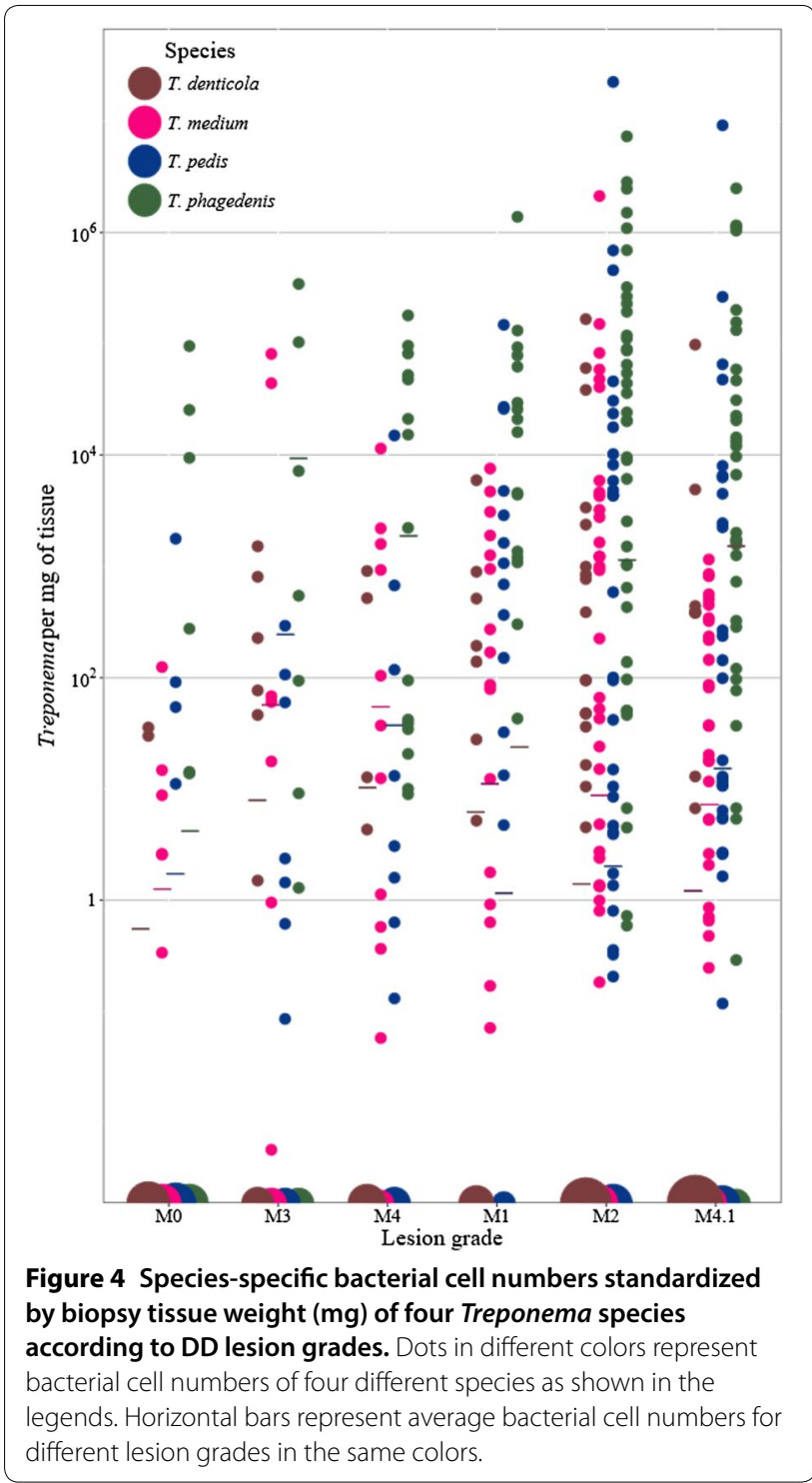

(69.2\%) followed by no species and T. denticola and $T$. pedis together which were equally likely at $15.4 \%$ (Figure 3). Most M0 samples did not contain any species of the 4 most common species of Treponema (74.2\%) and those that did contained a single species of T. phagedenis, T. medium, T. pedis or T. denticola $(9.7 \%, 3.2 \%, 6.5 \%$ and $6.5 \%$, respectively) (Figure 3). Finally, if $T$. denticola's presence or absence is ignored, T. medium, T. phagedenis, and T. pedis are found together in $54.1-72.5 \%$ of active lesions compared to $0 \%$ of M0 samples and M3 lesions and $33.3 \%$ of M4 lesions (Figure 3).

\section{Treponema abundance and species composition}

Absolute quantification of Treponema in biopsies by qPCR after weight standardization indicated the total number of Treponema increased in active lesions compared to chronic and healing DD lesions, regardless of species (Figure 4). Pairwise comparisons between the total number of Treponema between lesion grades indicated significant differences between active lesions, DDfree skin, and healing lesions. M1 lesions, M2 lesions, and M4.1 lesions had significantly more Treponema than M0 samples $(P<0.001,<0.001$, and $=0.01$, respectively $)$ by $4.2,3.8$ and 2.6 times, respectively (Figure 4 ). M1 lesions and M2 contained significantly more Treponema than M3 lesions ( $P=0.01$ and 0.009 , respectively) by 3.4 and 3.0 times, respectively (Figure 4 ).

Counts of T. phagedenis per mg of tissue were significantly higher than T. denticola, T. medium, and T. pedis $(P<0.001)$. Counts of $T$. denticola were significantly $(1.63$ times) lower than T. medium and T. pedis regardless of lesion grade $(P<0.001)$. There were no significant differences between T. medium and T. pedis counts irrespective of lesion grade $(P>0.99)$. Treponema counts between species indicated T. phagedenis and T. medium were the most correlated $\left(R^{2}=0.71\right)$, followed by $T$. medium and T. pedis $\left(\mathrm{R}^{2}=0.49\right)$ and T. phagedenis and T. pedis $\left(R^{2}=0.49\right)$. The amount of $T$. denticola was weakly correlated with $T$. medium, $T$. phagedenis and $T$. pedis $\left(R^{2}=0.40,0.32\right.$, and 0.30 , respectively) indicating counts of T. medium, T. pedis and T. phagedenis have little effect on $T$. denticola counts and vice versa.

\section{Species composition: direct absolute quantification of Treponema species in biopsies}

A multivariate analysis compared the number of an individual Treponema species according to lesion grade. Quantities (Treponema/mg of tissue) of T. phagedenis and $T$. pedis are highest in M2 lesions, M1 lesions and M4.1 lesions and lowest in M0 samples, M3 lesions, and M4 lesions (Figure 3). Quantities of T. phagedenis per mg of tissue were significantly higher in M1 lesions, M2 lesions, M4 lesions, and M4.1 lesions compared to M0 
samples $(P<0.001)$ but were not significantly different in M3 lesions $(P=0.97)$ compared to M0 samples (Figure 4) (Table 6). Quantities of T. pedis were significantly higher in M1 lesions and M2 lesions compared to M0 samples ( $P=0.018$ and 0.052 , respectively); however, $T$. pedis was not significantly higher in M4.1 lesions compared to M0 samples $(P=0.12)$ (Table 6). M1 lesions contained significantly more $T$. pedis than M3 lesions $(P=0.05)$ (Table 6). Similarly, T. medium was present in significantly higher quantities per mg of tissue in M1 lesions and M2 lesions compared to M0 samples $(P=0.02$ and 0.003 , respectively) (Table 6). However, there was no significant difference between $T$. medium counts in M4.1 lesions, M4 lesions, and M3 lesions and M0 samples $(P=0.32,0.47$, and 0.67 , respectively). Finally, $T$. denticola abundance per mg of biopsy tissue were not significantly different between lesion grades (Table 6).

\section{Discussion}

To our knowledge, this is the first study examining the distribution and absolute quantities of Treponema species among DD lesion grades. From the results presented here, we suggest that the four most common species of Treponema found in digital dermatitis, T. phagedenis, $T$. pedis, T. medium and T. denticola, can be identified at the species level using species-specific genes. Our qPCR results and validation demonstrate Treponema species can be identified in DD-lesion tissue at the species level in a single reaction, which may improve current prevalence estimates as a consistent, reliable way to identify species as opposed to phylotyping. We have found the number of Treponema species and their absolute quantities are higher in active lesions than in healing, chronic or DD-free skin suggesting Treponema abundance may influence host pathogenicity. Further, we have found strong relationships between the presence and absolute quantities of $T$. phagedenis, T. pedis and T. medium that are independent of $T$. denticola, suggesting there may be interactions between Treponema species found in DD.

We performed microbiome analysis on biopsies to validate our $\mathrm{qPCR}$ and look for additional species of Treponema. Our microbiome analysis results are consistent with previous studies indicating T. phagedenis, $T$. pedis, T. medium, and T. denticola are the most prominent species within lesions and are the most consistently isolated [9, 37]. The species-specific qPCR confirms that the four species of Treponema are consistently identified in DD lesions. The variation in Treponema abundance may have important implications to DD lesion scoring as well as host pathology. We found T. phagedenis is present in all lesions grades and typically the most readily isolated species, consistent with previous literature [19,
38]. Our results show $T$. denticola was identified the least frequently and with the lowest average copy numbers, but in some studies it is one of the most frequent with T. phagedenis [39]. Further, T. pedis was the third most common species identified and very close to T. medium; previous studies have found it is either identified in very low amounts [37] or high [5]. The second most common species was $T$. medium; previous studies have found $T$. medium-like to be among the most prevalent species and seeing as it is typically clustered with $T$. vincentii, which we found in very low amounts, these results are comparable [5, 37]. We did not find T. brennaborense or $T$. socranski, or any closely related ASVs, in our microbiome analysis and did not investigate them further. However, many studies have found T. brennaborense in DD lesions in relatively low amounts and a geographical component or presence as a fecal contaminant has been suggested to account for this discrepancy [1, 40-42]. These findings help resolve interactions between species that were previously unnoticed because T. pedis and/or T. putidum and $T$. denticola were clustered into a single phylotype ( $T$. denticola/T. putidum/T. pedis-like) [9, 40, 41].

Our species-specific qPCR also demonstrated that the presence and absolute quantities of Treponema species are correlated with one another irrespective of lesion grade such that the presence of certain species increase the odds of finding another and their absolute abundance tend to increase and decrease collectively. The likelihood of finding T. pedis, T. medium and T. phagedenis together is much higher than finding them apart. The presence or absence of the above-mentioned species have considerable predictive power on the presence or absence of the others. Additionally, there is a positive correlation between the quantities of the above-mentioned species with one another that if one increases the other two tend to increase as well. The presence or absence of one species is a better predictor of the presence or absence of another species than their absolute values suggesting interactions between species may not be strictly density dependent. However, there is weak to no correlations between the quantities and presence or absence of $T$. denticola with T. phagedenis, T. pedis, and T. medium.

The designations of active, chronic, healing and DDfree heel bulb groupings are related to the total number of Treponema cells (abundance). We found the total number of Treponema cells were highest in M1 lesions, M2 lesions, and M4.1 lesions and lowest in M0 samples, M3 lesions, and M4 lesions. Previous studies have suggested that due to the high variation of phylotypes identified within lesions between studies, Treponema abundance may have a greater effect on pathology than species diversity [41]. Krull et al. [19] found Treponema diversity increased following treatment with tetracycline and 
Table 6 Difference between the natural logarithm of abundance (copies/mg of tissue) of Treponema species across lesion grades

\begin{tabular}{|c|c|c|c|c|c|}
\hline \multirow[t]{2}{*}{ Lesion comparison } & \multicolumn{4}{|l|}{ Species } & \multirow[t]{2}{*}{ Total } \\
\hline & T. denticola & T. medium & T.pedis & T.phagedenis & \\
\hline M0-M1 & -2.07 & $-3.70^{* *}$ & $-4.44^{* * *}$ & $-6.30^{* * *}$ & $-4.20^{* * *}$ \\
\hline M0-M2 & 1.97 & $-3.72^{* * *}$ & $4.21^{\ddagger}$ & $-6.75^{* * *}$ & $-3.82^{* * *}$ \\
\hline M0-M3 & -1.91 & 1.67 & -3.20 & -4.77 & -0.84 \\
\hline M0-M4 & 1.22 & -2.02 & 3.76 & $5.13^{* *}$ & -2.07 \\
\hline M0-M4.1 & 1.80 & -2.17 & -2.90 & $5.58^{* * *}$ & $-2.64^{*}$ \\
\hline M1-M2 & 1.33 & 1.83 & 2.97 & -4.69 & 0.39 \\
\hline M1-M3 & 1.06 & 1.70 & $2.52^{*}$ & $-3.60^{* * *}$ & $3.36^{* *}$ \\
\hline $\mathrm{M} 1-\mathrm{M} 4$ & 1.16 & 1.53 & $-2.68^{\ddagger}$ & -3.52 & 2.14 \\
\hline M1-M4.1 & -0.84 & -1.86 & -2.23 & 1.53 & 1.57 \\
\hline M2-M3 & -0.74 & 1.86 & 1.53 & $1.98^{* * *}$ & $2.98^{* * *}$ \\
\hline $\mathrm{M} 2-\mathrm{M} 4$ & -0.74 & 1.56 & 1.24 & 2.07 & 1.75 \\
\hline M2-M4.1 & -0.64 & -0.30 & -0.68 & 1.62 & 1.18 \\
\hline M3-M4 & -0.16 & -0.16 & 0.29 & -1.17 & -1.23 \\
\hline M3-M4.1 & -0.10 & 0.14 & -0.45 & $0.45^{\ddagger}$ & -1.79 \\
\hline M4-M4.1 & -0.10 & 0.02 & -0.23 & -0.08 & -0.57 \\
\hline
\end{tabular}

Negative values indicate the mean log abundance was that amount less the lesion grade on the right; positive values indicate the mean log abundance was that amount greater than the lesion grade on the right. Values represent the difference in species abundance between lesion grades (e.g. T. denticola is 2.07 times less abundant in M0s compared to M1s).

${ }^{*} P<0.05,{ }^{* *} P<0.01,{ }^{* * *} P<0.001,{ }^{*} 0.051<P<0.9$.

T. denticola was more abundant relative to active lesions grades. While we did not notice a significant increase in T. denticola in M3 samples, they were consistently among the only Treponema still viable by culture following lesion treatment with tetracycline. This difference may be due to a decreased time allotted between treatment and sampling. There was no difference between Treponema abundance in M0 samples, M3 lesions and M4 lesions; if healing lesions have approximately equal Treponema abundances to chronic lesions this may provide an insight into the chronic, cyclical nature of DD lesions.

Both the total number of Treponema cells and the number of Treponema species increases in active lesions compared to chronic, healing or DD-free skin. We found the number of Treponema species is most similar between M0 samples and M3 lesions, M4 lesions and M1 lesions, and M4.1 lesions and M2 lesions where larger, active lesions have higher numbers of Treponema, chronic and small active lesions have intermediate, and healing and DD-free have the fewest. Because most active lesions contain T. phagedenis, T. pedis, and T. medium and our results suggest these species are not significantly correlated with $T$. denticola, it is possible the pathogenicity of Treponema associated with DD is related to interactions between these three species regardless of $T$. denticola. The number of $T$. denticola cells isolated does not differ significantly between lesions grades, including M0.
Furthermore, most biopsies do not contain T. denticola, ranging from 40 to $94 \%$ over all lesion grades except for M3 lesions, supporting a negative association between macroscopic signs of pathogenicity and the presence of $T$. denticola. Interestingly, the most significant increase in Treponema abundance between lesions grades at the species level were T. pedis and T. phagedenis from healthy to active lesions. Further, the macroscopic changes associated with transitioning from active to a healing lesions are accompanied by a decrease in $T$. pedis abundance. While T. phagedenis is present throughout grades of DD, T. pedis and T. phagedenis counts are significantly higher and correlated to one another in active, but not in chronic lesions, suggesting pathogenicity associated with active lesions may be related to interactions between these two species.

If we ignore potential interactions among species of Treponema isolated from DD lesions, there are significant differences in species presence or absence between lesion grades when species are treated independently. $T$. phagedenis and T. pedis are significantly more likely to be found in active lesions than healthy, healing, and chronic DD grades, supporting a relationship between host ulcerative pathology and both the presence and amount of $T$. pedis and T. phagedenis. The likelihood of $T$. denticola presence does not increase in any lesion in comparison with M0 except for M3 lesions, providing further 
indication T. denticola may not be related to the characteristic pathology associated with active DD lesions. Taken together, the absence of an association among $T$. denticola and the other three Treponema species, insignificant changes in $T$. denticola abundance between lesions grades, and $T$. denticola abundance increasing while the other 3 species of Treponema decrease following antibiotic treatment, may also suggest negative associations between T. denticola and T. phagedenis, T. pedis and T. medium.

Increasing Treponema spp. diversity from healing to chronic to active DD lesions is supported by deepsequencing findings in Krull et al. [19]. However, when lesions are grouped based on the single most common species composition, M1 M4, and M4.1 lesions have the same most common species composition ( $\mathrm{Ph} / \mathrm{Me} / \mathrm{Pe})$ whereas all four species $(\mathrm{Ph} / \mathrm{Me} / \mathrm{Pe} / \mathrm{De})$ are most likely to be found together in M2 lesions, suggesting the most common species composition is not the same within all active lesions (M1s and M4.1s are more similar in composition to a chronic lesion (M4) than an advanced, active lesion (M2). Similarly, M3 lesions and M0 samples have distinct species compositions which is likely due to treatment with tetracycline selecting against some, but not all, species. Further, lesions following antibiotic treatment are more similar in species profile to M0 samples than active or chronic lesions but not significantly different in terms of Treponema cell count, suggesting treatment decreases Treponema diversity but not Treponema cell count. However, culture results suggest $T$. denticola and to a lesser extent $T$. phagedenis, remain viable after treatment with tetracycline unlike T. pedis and T. medium. In vitro minimum bactericidal concentrations (MBCs) of $T$. denticola-like DD isolates is between 3 and $6 \mathrm{mg} / \mathrm{L}$ and is not a recommended antibiotic to treat periodontal infections with T. denticola [7, 43]. Conversely, MBCs for T. phagedenis-like and T. medium/T. vincentii-like groups are $1.5-6 \mathrm{mg} / \mathrm{L}$ and $0.75 \mathrm{mg} / \mathrm{L}$, respectively, supporting our culture viability findings following tetracycline treatment and suggesting $T$. denticola may be more resistant to treatment with oxytetracycline than $T$. medium, $T$. pedis and T. phagedenis [7]. Taken together, our findings and previous literature suggest $T$. denticola has a smaller impact on host ulcerative pathology than T. phagedenis, T. pedis and T. medium but may resist antibiotic treatment, facilitating chronic infection states that allow subsequent lesion recolonization of susceptible species with greater influences on host pathogenicity.

The results presented here suggest there is a relationship between the macroscopic morphological changes between DD lesion grades and species composition, individual species abundances, and total Treponema abundance. While these results represent a subset of the Treponema species present in DD lesions, and it is possible the nature of these interactions is much more intricate and complex, the four species analyzed here are consistently the most prevalent and abundant in DD globally. We have demonstrated species of Treponema in DD can be identified based on species-specific genes and believe future research will be greatly enhanced by determining species of Treponema precisely. Many researchers are currently sequencing Treponema genomes which will allow currently unidentified species to be identified, potentially using species-specific genes, and contribute to future research analyzing correlations between species and morphological shifts of DD lesions. Our results support a significant role of Treponema in macroscopic pathology of DD lesions; however, because many bacteria are consistently found in addition to Treponema, we believe future research would benefit from analyzing additional genera of bacteria.

\section{Additional file}

Additional file 1. Description of the samples included in the microbiome analysis.

\section{Authors' contributions}

$\mathrm{CB}$ and JDB designed protocols and hypotheses. $\mathrm{KO}$ demonstrated sampling techniques on farm. CB conducted animal sampling under protocol created by $\mathrm{KO}$ with certified hoof-trimmers for Alberta farms and Abattoir and CL conducted sampling in Saskatchewan. CB cultured and isolated Treponema from biopsies and designed and species-specific PPCR and PCR with genes identified by SN. EK and HD performed microbiome analysis and algorithm for GPCR validation. CB and SAN designed statistical analysis and figures generated by SAN. Manuscript written by CB and JDB, and edited and reviewed by KO, SAN, $\mathrm{SN}, \mathrm{CL}, \mathrm{EK}$, and HD. All authors read and approved the final manuscript.

\section{Author details}

${ }^{1}$ Department of Production Animal Health, Faculty of Veterinary Medicine, University of Calgary, Calgary, AB, Canada. ${ }^{2}$ Department of Large Animal Clinical Sciences, Western College of Veterinary Medicine, University of Saskatchewan, Saskatoon, SK, Canada. ${ }^{3}$ Department of Animal Science, Faculty of Agricultural and Food Sciences, University of Manitoba, Winnipeg, MB, Canada.

\section{Acknowledgements}

We thank the Alberta dairy producers for their support and continued participation in UCVM research. We would like to thank Alberta hoof-trimmers Elbert Koster (No-Tilt Hoof Trimming, Alberta) and Rob Geier (Casper Hoof Trimming Ltd.) for their support, assistance, knowledge, and for contacting producers. We thank Dr. Döpfer for her Treponema DNA isolated from bovine DD that contributed to the identification of species-specific genes.

\section{Author details \\ ${ }^{1}$ Department of Production Animal Health, Faculty of Veterinary Medicine, University of Calgary, Calgary, AB, Canada. ${ }^{2}$ Department of Large Animal Clini- cal Sciences, Western College of Veterinary Medicine, University of Saskatch- ewan, Saskatoon, SK, Canada. ${ }^{3}$ Department of Animal Science, Faculty of Agri- cultural and Food Sciences, University of Manitoba, Winnipeg, MB, Canada.}

\section{Funding}

Research funding provided by Alberta Agriculture and Forestry, CB was funded by Natural Sciences and Engineering Research Council (NSERC), SAN 
by Canadian Bovine Mastitis and Milk Quality Research Network (CBMQRN), and SN by CBMQRN and the Eyes High program at the University of Calgary.

\section{Publisher's Note}

Springer Nature remains neutral with regard to jurisdictional claims in published maps and institutional affiliations.

Received: 20 July 2018 Accepted: 11 September 2018 Published online: 29 October 2018

\section{References}

1. Wilson-Welder J, Alt DP, Nally JE (2015) Digital dermatitis in cattle: current bacterial and immunological findings. Animals 5:1114-1135

2. Cook NB (2016) LifeStep - a lesion oriented, life cycle approach to preventing lameness in dairy herds. TEMA: Koen og kalven KvægKongres 2016, pp 1-11

3. Underwood WJ, Blauwiekel R, Delano ML, Gillesby R, Mischler SA, Schoell A (2015) Chapter 15: biology and diseases of ruminants (Sheep, Goats, and Cattle). In: Laboratory animal medicine, $3^{\text {rd }}$ edition. Elsevier, pp 623-694

4. Solano L, Barkema HW, Jacobs C, Orsel K (2017) Validation of the $\mathrm{M}$-stage scoring system for digital dermatitis on dairy cows in the milking parlor. J Dairy Sci 100:1592-1603

5. Evans NJ, Brown JM, Demirkan I, Singh P, Getty B, Timofte D, Vink WD, Murray RD, Blowey RW, Birtles RJ, Hart CA, Carter SD (2009) Association of unique, isolated treponemes with bovine digital dermatitis lesions. J Clin Microbiol 47:689-696

6. Plummer PJ, Krull A (2017) Clinical perspectives of digital dermatitis in dairy and beef cattle. Vet Clin North Am Food Anim Pract 33:165-181

7. Evans NJ, Brown JM, Demirkan I, Birtles R, Hart CA, Carter SD (2009) In vitro susceptibility of bovine digital dermatitis associated spirochaetes to antimicrobial agents. Vet Microbiol 136:115-120

8. Zinicola M, Lima F, Lima S, Machado V, Gomez M, Döpfer D, Guard C, Bicalho R (2015) Altered microbiomes in bovine digital dermatitis lesions, and the gut as a pathogen reservoir. PLoS One 10:e0120504

9. Evans NJ, Brown JM, Demirkan I, Murray RD, Vink WD, Blowey RW, Hart CA, Carter SD (2008) Three unique groups of spirochetes isolated from digital dermatitis lesions in UK cattle. Vet Microbiol 130:141-150

10. Nielsen MW, Strube ML, Isbrand A, Al-Medrasi WDHM, Boye M, Jensen TK, Klitgaard K (2016) Potential bacterial core species associated with digital dermatitis in cattle herds identified by molecular profiling of interdigital skin samples. Vet Microbiol 186:139-149

11. Gomez A, Cook NB, Bernardoni ND, Rieman J, Dusick AF, Harthorn R, Socha MT, Read DH, Döper D (2012) An experimental infection model to induce digital dermatitis infection in cattle. J Dairy Sci 95:1821-1830

12. Krull AC, Cooper VL, Coatney JW, Shearer JK, Gorden PJ, Plummer PJ (2016) A highly effective protocol for the rapid and consistent induction of Digital Dermatitis in Holstein calves. PLoS One 11:e0154481

13. Döpfer D, Koopmans A, Meijer FA, Szakall I, Schukken YH, Klee W, Bosma RB, Cornelisse JL, van Asten AJAM, ter Huurne AAHM (1997) Histological and bacteriological evaluation of digital dermatitis in cattle, with special reference to spirochaetes and Campylobacter faecalis. Vet Rec 140:620-623

14. Murray RD, Carter SD (2015) Bovine digital dermatitis: current concepts from laboratory to farm. Vet J 211:3-13

15. Döpfer D, Anklam K, Mikheil D, Ladell P (2012) Growth curves and morphology of three Treponema subtypes isolated from digital dermatitis in cattle. Vet J 193:685-693

16. Angell JW, Clegg SR, Sullivan LE, Ducan JS, Grove-White DH, Carter SD, Evans NJ (2015) In vitro susceptibility of contagious ovine digital dermatitis associated Treponema spp. isolates to antimicrobial agents in the UK. Vet Dermatol 26:484-487

17. Stamm LV, Bergen HL, Walker RL (2002) Molecular typing of papillomatous dermatitis-associated Treponema analysis of 16S-23S ribosomal DNA intergenic spacer regions. J Clin Microbiol 40:3463-3469

18. Pringle $M$, Bergsten $C$, Fernström LL, Höök H, Johansson KE (2008) Isolation and characterization of Treponema phagedenis-like spirochetes from digital dermatitis lesions in Swedish dairy cattle. Acta Vet Scand 50:40
19. Krull AC, Shearer JK, Gorden PJ, Cooper VL, Philips GJ, Plummer PJ (2014) Deep sequencing analysis reveals temporal microbiota changes associated with development of bovine digital dermatitis. Infect Immun 82:3359-3373

20. Orsel K, Plummer P, Shearer J, De Buck J, Carter SD, Guatteo R, Barkema HW (2018) Missing pieces of the puzzle to effectively control digital dermatitis. Transbound Emerg Dis 1:186-198

21. Berry SL, Read DH, Famula TR, Mongini A, Döpfer D (2012) Long-term observations on the dynamics of bovine digital dermatitis lesions on a California dairy after topical treatment with lincomycin $\mathrm{HCl}$. Vet J 193:654-658

22. Radolf JD (1996) Treponema. Medical microbiology, 4th edn. University of Texas Medical Branch at Galveston, Galveston (TX) Chapter 36

23. Naushad S, Barkema HW, Luby C, Condas LAZ, Nobrega DB, Carson DA, De Buck J (2016) Comprehensive phylogenetic analysis of bovine non-aureus Staphylococci species based on whole-genome sequencing. Front Microbiol 7:1990

24. Pruitt KD, Tatusova T, Maglott DR (2005) NCBI Reference Sequence (RefSeq): a curated non-redundant sequence database of genomes, transcripts and proteins. Nucleic Acids Res 33:D501-D504

25. Markowitz VM, Chen I-MA, Palaniappan K, Chu K, Szeto E, Grechkin Y, Ratmer A, Jacob B, Huang J, Williams P, Huntermann M, Anderson I, Mavromatis K, Ivanova NN, Kyrpides NC (2012) IMG: the integrated microbial genomes database and comparative analysis system. Nucleic Acids Res 40:115-122

26. Wattam AR, Abraham D, Dalay O, Disz T, Driscoll T, Gabbard J, Gillespie J, Gough R, Hix D, Kenyon R, MacHi D, Mao C, Nordberg E, Olson R, Overbeek R, Pusch G, Shukla M, Schulman J, Stevens R, Sullivan D, Vonstein V, Warren A, Will R, Wilson M, Yoo H, Zhang C, Zhang Y, Sobral B (2014) PAT$\mathrm{RIC}$, the bacterial bioinformatics database and analysis resource. Nucleic Acids Res 42:D581-D591

27. Yoon S-H, Ha S-M, Kwon S, Lim J, Kim Y, Seo H, Chun J (2017) Introducing EzBioCloud: a taxonomically united database of 165 rRNA gene sequences and whole-genome assemblies. Int J Syst Evol Microbiol 67:1613-1617

28. Naushad HS, Lee B, Gupta RS (2014) Conserved signature indels and signature proteins as novel tools for understanding microbial phylogeny and systematics: identification of molecular signatures that are specific for the phytopathogenic genera Dickeya, Pectobacterium and Brenneria. Int J Syst Evol Microbiol 64:366-383

29. Shen Z, Qu W, Wang W, Lu Y, Wu Y, Li Z, Hang X, Wand X, Zhao D, Zhang C (2010) MPprimer: a program for reliable multiplex PCR primer design. BMC Bioinformatics 11:143

30. Edgar RC (2016) UNOISE2: improved error-correction for Illumina 165 and ITS amplicon sequencing. bioRxiv 081257

31. Edgar RC (2013) UPARSE: highly accurate OTU sequences from microbial amplicon reads. Nat Methods 10:996-998. https://doi.org/10.1038/nmeth .2604

32. DeSantis TZ, Hugenholtz P, Larsen N, Rojas M, Brodie EL, Keller K, Huber T, Dalevi D, Hu P, Andersen GL (2006) Greengenes, a chimera-checked 165 rRNA gene database and workbench compatible with ARB. Appl Environ Microbiol 72:5069-5072

33. Plaire D, Puaud S, Marsolier-Kergoat M-C, Elalouf J-M (2017) Comparative analysis of the sensitivity of metagenomic sequencing and PCR to detect a biowarfare simulant (Bacillus atrophaeus) in soil samples. PLoS One 12:e0177112

34. Smith TF, Waterman MS (1981) Identification of common molecular subsequences. J Mol Biol 147:195-197

35. Gandrud C (2017) Easily Install and Load Stable Zelig Packages [R package zeligverse version 0.1.1]

36. Ripley B, Venables W, Maintainer (2016) Package "nnet" feed-forward neural networks and multinomial log-linear models 14:52:39 https:// www.r-project.org/

37. Klitgaard K, Bretó AF, Boye M, Jensen TK (2013) Targeting the treponemal microbiome of digital dermatitis infections by high-resolution phylogenetic analyses and comparison with fluorescent in situ hybridization. J Clin Microbiol 51:2212-2219

38. Trott DJ, Moeller MR, Zuerner RL, Goff J, Waters W, Alt D, Richard L, Wannemuehler M, Walker R (2003) Characterization of Treponema phagedenislike spirochetes isolated from papillomatous digital dermatitis lesions in dairy cattle. J Clin Microbiol 41:2522-2529 
39. Yano T, Moe KK, Chuma T, Misawa N (2010) Antimicrobial susceptibility of Treponema phagedenis-like spirochetes isolated from dairy cattle with papillomatous digital dermatitis lesions in Japan. J Vet Med Sci 72:379-382

40. Klitgaard K, Boye M, Capion N, Jensen TK (2008) Evidence of multiple Treponema phylotypes involved in bovine digital dermatitis as shown by 165 rRNA gene analysis and fluorescence in situ hybridization. J Clin Microbiol 46:3012-3020

41. Rasmussen M, Capion N, Klitgaard K, Rogdo T, Fjeldaas T, Boye M, Jensen T (2012) Bovine digital dermatitis: possible pathogenic consortium consisting of Dichelobacter nodosus and multiple Treponema species. Vet Microbiol 160:151-161

42. Evans NJ, Brown JM, Murray RD, Getty B, Birtles R, Hart C, Carter SD (2011) Characterization of novel bovine gastrointestinal tract Treponema isolates and comparison with bovine digital dermatitis treponemes. Appl Environ Microbiol 77:138-147

43. Hardham JM, Rosey EL (2000) Antibiotic selective markers and spirochete genetics. J Mol Microbiol Biotechnol 2:425-432
Ready to submit your research? Choose BMC and benefit from:

- fast, convenient online submission

- thorough peer review by experienced researchers in your field

- rapid publication on acceptance

- support for research data, including large and complex data types

- gold Open Access which fosters wider collaboration and increased citations

- maximum visibility for your research: over $100 \mathrm{M}$ website views per year

At BMC, research is always in progress.

Learn more biomedcentral.com/submissions 